Pacific Journal of Mathematics

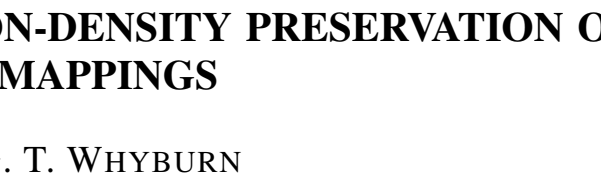




\title{
DIMENSION AND NON-DENSITY PRESERVATION OF MAPPINGS
}

\author{
G. T. WHYBURN
}

1. Introduction. In this paper consideration is given to conditions under which the property of being non-dense in a space in the sense of containing no open set in that space is invariant under certain types of mappings. In some spaces and for some mapping types the issue involved is essentially equivalent to the question of dimensionality preservation. These questions are of interest and importance in numerous mathematical fields. They are especially so in the study of topological aspects of the theory of functions and it is toward this connection that the results and methods in this note will be largely directed.

A single valued continuous transformation $f(X)=Y$ will be called a mapping. Such a mapping is open if open sets in $X$ have open images in $Y$ and is light provided $f^{-1}(y)$ is totally disconnected for each $y \in Y$. Also $f$ has scattered point inverses provided that for each $y \in Y, f^{-1}(y)$ is a scattered set in the sense that no point of $f^{-1}(y)$ is a limit point of $f^{-1}(y)$.

As indicated above, a set $K$ in a space $X$ is non-dense in $X$ provided $K$ contains no open set in $X$. On the other hand that $K$ is dense in $X$ means that every point of $X$ is either a point or a limit point of $K$. A mapping $f(X)=Y$ is said to preserve non-density for compact sets provided that $f(K)$ is non-dense in $Y$ whenever $K$ is compact and non-dense in $X$. For a mapping $f(X)=Y$, a subset $X_{0}$ of $X$ is said to be semidense in $X$ provided $X_{0}$ is dense in some open subset of every open set $U$ in $X$ whose image $f(U)$ is also open in $Y$. Thus the property of semi-density is a property of a subset of $X$ relative to a mapping $f$ on $X$ and not an intrinsic property of $X_{0}$ alone.

For a mapping $f(X)=Y$, the set of all $x \in X$ such that $x$ is a component of $f^{-1} f(x)$ will be designated by the symbol $D_{f}$. Also the symbol $L_{f}$ will be used for the set of all $x \in X$ such that $f^{-1} f(x)$ is totally disconnected. Thus $L_{f}$ is the maximum inverse set in $X$ on which the mapping $f$ is light, where by an inverse set $I$ we mean a set which is the inverse of its transform under $f$, that is, one satisfying the relation

$$
I=f^{-1} f(I) \text {. }
$$

Accordingly $L_{f}$ may be thought of as the lightness kernel or 0-dimensional kernel of the mapping $f$. Obviously we have $L_{f} \subset D_{f}$. 
2. General setting. We begin with a theorem which was suggested by the theorem of Alexandroff's [1] on invariance of dimension under countable-fold open mappings. Our proof closely parallels that of Alexandroff for his theorem.

(2.1) THEOREM. Let $f(A)=B$ be open and have scattered point inverses, where $A$ and $B$ are locally compact separable and metric. Then $A$ is the union $A=\sum A_{n}$ of a sequence of compact sets such that $f \mid A_{n}$ is topological for each $n$.

Proof. Let $\left(U_{n}\right)$ be a countable basis of open sets in $A$, so chosen that $\bar{U}_{n}$ is compact for each $n$. For each $n$, let $F_{n}$ be the set of all $x \in U_{n}$ such that $g_{n}^{-1} g_{n}(x)=x$ where $g_{n}$ denotes the mapping $f \mid U_{n}$. Then $F_{n}$ is closed in $U_{n}$ by openness of $f$. Accordingly each $F_{n}$ is the union of a countable sequence of compact sets and thus we can write $\sum F_{n}=$ $\sum A_{n}$ where each $A_{n}$ is compact and lies in some $F_{m}$. Thus $f \mid A_{n}$ is topological for each $n$. Finally, $\sum A_{n}=A$, because if $x \in A$, there exists an $m$ such that $x \in U_{m}$ and $U_{m} \cdot f^{-1} f(x)=x$ and hence so that $x \in F_{m} \subset$ $\sum F_{n}=\sum A_{n}$.

(2.11) Corollary. For any closed set $K$ in $A$ we have

$$
\operatorname{dim} f(K)=\operatorname{dim} K \text {. }
$$

(2.12) Corollary. If $K$ is any closed set in $A$ and $V$ is any open subset of $f(K)$, then $V$ contains an open subset $U$ which is homeomorphic with a subset of $K$.

For let $K_{n}$ denote the set $K \cdot A_{n}$ for each $n$. Then since $V \subset \sum f\left(K_{n}\right)$ and each $f\left(K_{n}\right)$ is compact, some $f\left(K_{n}\right)$ contains an open subset $U$ of $V$. Then $K_{n} \cdot f^{-1}(U)$ maps topologically onto $U$ under $f$.

(2.13) CoRollary. If $A$ and $B$ are 2-manifolds, (or n-manifolds), then if $K$ is non-dense in $A, f(K)$ is non-dense in $B$.

(2.2) Theorem. Let $A$ and $B$ be locally compact separable metric spaces and let $f(A)=B$ be a mapping preserving compact non-dense sets. Then for some $y \in B, f^{-1}(y)$ is totally disconnected.

Proof. For each integer $n>0$ and each $x \in A$, let $U_{x}^{n}$ be an open set of diameter $<1 / n$ containing $x$ and having a compact boundary $F_{x}^{n}$. Let $U_{x_{1}}^{n}, U_{x_{2}}^{n}, \cdots$ be a countable collection of these sets $U_{x}^{n}$ whose union covers $A$ and set

$$
F^{n}=\sum_{i} F_{x_{i}}^{n}, \quad F=\sum_{n} F^{n}=\sum_{n} \sum_{i} F_{x_{i}}^{n} .
$$


Now $f(F) \neq B$. For if $f(F)=B$, then for some $n$ and $i$ the set $f\left(F_{x_{i}}^{n}\right)$ must contain an open set in $B$, as $B$ is locally compact; and this is impossible by hypothesis because $F_{x_{i}}^{n}$ is compact and non-dense for each $n$ and $i$. Accordingly there exists a $y \in B-f(F)$. Clearly $f^{-1}(y)$ is totally disconnected, because if it had a non-degenerate component $C_{y}$, then for $1 / n<$ the diameter of $C_{y}$, we would have $C_{y} \cdot F_{x_{i}}^{n} \neq 0$ where $C_{y} \cdot U_{x_{i}}^{n} \neq 0$.

(2.21) Corollary. Under the same hypothesis, the set $Y$ of all $y \in B$ with $f^{-1}(y)$ totally disconnected is dense in $B$ and $L_{f}=f^{-1}(Y)$ is semi-dense in $A$.

For if $B_{0}$ is any open set in $B$, we have only to set $A_{0}=f^{-1}\left(B_{0}\right)$ and apply the theorem to the mapping $f \mid A_{0}$ to obtain the first conclusion that $Y$ is dense in $B$. To prove the second conclusion suppose on the contrary that for an open set $U$ which has an open image and which we first suppose conditionally compact, $L_{f} \cdot U$ is dense in no open subset of $U$. Then $\overline{L_{f} \cdot U}$ is compact and non-dense, whereas $f\left(\overline{L_{f} \cdot U}\right)$ must contain $f(U)$ since $Y$ is dense in $f(U)$ by openness of $f(U)$. This is a contradiction.

Finally, to see that $U$ need not be conditionally compact, we need only show that any open set $V$ in $A$ with an open image contains a conditionally compact open subset $U$ with an open image. To do this, set $V=\sum V_{n}$ where each $V_{n}$ is open and conditionally compact and $\bar{V}_{n} \subset U$. Then since $f(V)=\sum f\left(\bar{V}_{n}\right)$, some $f\left(\bar{V}_{n}\right)$ contains an open set $G$. Since $f\left[\operatorname{Fr}\left(V_{n}\right)\right]$ is non-dense, $Q=G-f\left[\operatorname{Fr}\left(V_{n}\right)\right]$ is open and nonempty. Then $U=V_{n} \cdot f^{-1}(Q)$ meets our condition.

(2.3) Theorem. Let $A$ and $B$ be locally compact separable metric spaces with $\operatorname{dim} A=k<\infty$ and let $f(A)=B$ be a mapping such that the image of every compact non-dense set $K$ with $\operatorname{dim} K<k$ is non-dense. Then the set $Y$ of all $y \in B$ with $f^{-1}(y)$ totally disconnected is dense in $B$.

For, in the preceding proofs the sets $F_{x}^{n}$ could now be taken of dimension $\leqq k-1$.

3. Quasi-open mappings. Region on a sphere. A mapping $f(X)=$ $Y$ is quasi-open provided that if $y \in Y$ and $K$ is a compact component of $f^{-1}(y)$, then for any open set $U$ in $X$ containing $K, y$ is interior to $f(U)$ rel. $Y$, and is strongly quasi-open provided $y$ is interior to $f(U)$ relative to a larger space $Y_{0} \supset Y$. A mapping $f(X)=Y$ is monotone provided $f^{-1}(y)$ is a continuum (compact and connected set) for each $y \in Y$; and $f$ is compact provided $f^{-1}(K)$ is compact for every compact 
set $K \subset Y$ or, equivalently, provided $f$ is closed and has compact point inverses. For compact mappings, quasi-openness is equivalent to quasimonotoneity as defined originally by Wallace [3].

(3.1) THEOREM. Let $f(X)=Y$ be a compact and quasi-open mapping where $X$ is a region on a sphere $S, Y$ is a metric space and where no component of a point inverse separates $X$. In order that the image of every compact 1-dimensional set in $X$ be of dimension $\leqq 1$ it is necessary and sufficient that the set $D_{f}$ be semi-dense in $X$.

Proof. Let $f=l m, m(X)=X^{\prime}, l\left(X^{\prime}\right)=Y$ be the monotone-light factorization of $f$. Let the mapping $m$ be extended to the whole sphere $S$ by decomposing $S$ into the sets $m^{-1}\left(x^{\prime}\right), x^{\prime} \in X^{\prime}$ together with the components of $S-X$ so that we obtain a monotone mapping $\phi(S)=S^{\prime}$ of $S$ onto a sphere $S^{\prime}$ containing $X^{\prime}$ ( $\phi$ is the natural mapping of the decomposition) which is identical with $m$ on $X$. That $S^{\prime}$ is a topological sphere follows from the readily verified facts that the described decomposition of $S$ is upper semi-continuous and no element of this decomposition separates $S$, together with the classical theorem of R. L. Moore [2] that the hyperspace of any such decomposition of a sphere into continua is itself a topological sphere. Then $l\left(X^{\prime}\right)=Y$ is a light open mapping which is compact; and since $X^{\prime}$ is a region on $S^{\prime}, Y$ is a 2manifold by the invariance of the 2-manifold property under such mappings [4].

Now to prove the sufficiency of the condition let $K$ be a compact 1-dimensional set in $X$. Then $\operatorname{dim} m(K) \leqq 1$. For, if not, then $m(K)$ contains an open set $U$ in $X^{\prime}$. Then $l(U)$ is open in $Y$ and thus $m^{-1}(U)$ is an open set in $X$ whose image under $f$ is open in $Y$. Accordingly $D_{f}$ is dense in an open subset $Q$ of $m^{-1}(U)$. Since $Q$ cannot lie wholly in $K, Q-Q \cdot K$ contains a point $x$ of $D_{f}$. But then since $x=m^{-1} m(x)$, $m(x)$ cannot lie in $m(K)$, contrary to the supposition that $m(x) \in U \subset m(K)$. Thus $\operatorname{dim} m(K) \leqq 1$.

It remains to show that $\operatorname{dim} \operatorname{lm}(K) \leqq 1$. Since $l$ is compact, open and light and $X^{\prime}$ is a 2-manifold, $l$ is finite to one [4]. Hence by (2.11) we have $\operatorname{dim} \operatorname{lm}(K)=\operatorname{dim} m(K) \leqq 1$.

To prove the necessity of the condition we note first that it follows from our hypothesis that $f$ preserves non-density for compact sets. For if $K$ is a compact, non-dense set in $X$ we have $\operatorname{dim} K \leqq 1$. Whence $\operatorname{dim} f(K) \leqq 1$; and since as shown above $Y$ also is a 2-manifold, it follows from this that $f(K)$ is non-dense. Accordingly, by (2.21) not only $D_{f}$ but also $L_{f}$ must be semi-dense in $X$.

Clearly we have the following alternative form of (3.1) which we state as 
(3.2) Theorem. Let $f, X$ and $Y$ be as described in the first sentence of (3.1). In order that $f$ preserve non-density for compact sets it is necessary and sufficient that $L_{f}$ be semi-dense in $X$.

4. Quasi-open mappings on the general 2-manifold. We now show that the case of a mapping of this same type operating on an arbitrary 2-manifold can be reduced essentially to the case of a region on a sphere so that similar conclusions hold.

(4.1) Lemma. Let $f(X)=Y$ be quasi-open where $X$ is a 2-manifold without edges and $Y$ is a locally connected generalized continuum and suppose that $f\left(L_{f}\right)$ is dense in $Y$. If there exists in $X$ a compact set $K$ of dimension $\leqq 1$ whose image contains an open set in $Y$, then there exists a region $R$ in $X$ contained in a 2-cell of $X$ such that $Q=f(R)$ is open in $Y$, the mapping $f(R)=Q$ is compact and quasi-open and for some compact subset $K_{1}$ of $K \cdot R, f\left(K_{1}\right)$ contains an open set.

Proof. Let $V$ be an open set in $f(K)$. Then there is a point $y \in V$ such that $f^{-1}(y)$ is totally disconnected. Now for each $x \in K \cdot f^{-1}(y)$ there exists a 2-cell $E_{x}$ on $X$ with edge $C_{x}$ and interior $I_{x}$ such that $f\left(E_{x}\right) \subset V, C_{x} \cdot f^{-1}(y)=0$. Thus if $Q_{x}$ is the component of $Y-f\left(C_{x}\right)$ containing $y$ and $R_{x}$ is the component of $f^{-1}\left(Q_{x}\right)$ containing $x$ we have $R_{x} \subset I_{x}$ because $R_{x} \cdot C_{x}=0$. Accordingly, $R_{x}$ being conditionally compact [5], $f\left(R_{x}\right)=Q_{x} \subset V$ and the mapping $f\left(R_{x}\right)=Q_{x}$ is compact and quasiopen.

Now since $K \cdot f^{-1}(y)$ is covered by a finite union $U$ of the sets $R_{x}$ and $f(K \cdot U)$ contains an open set $V-f(K-K \cdot U)$ in $V$ about $y$, some one of the sets $R_{x}$, say $R$, is such that $f(K \cdot R)$ contains an open set. Since $K \cdot R$ is closed in $R$, for some compact set $K_{1} \subset K \cdot R, f\left(K_{1}\right)$ must likewise contain an open set in $Q=f(R)$. Thus the lemma is proven.

Since a region on a 2-cell may be considered as a region on a sphere (by mapping the 2-cell topologically onto a 2-cell on a sphere), this lemma together with the theorems in $\S 3$ yield at once

(4.2) THEOREM. Given a quasi-open mapping $f(X)=Y$ where $X$ is a 2-manifold without edges and $Y$ is a locally connected generalized continuum such that no component of a point inverse lying inside a closed 2-cell on $X$ separates $X$, in order that $f$ preserve non-density for compact sets it is necessary and sufficient that $L_{f}$ be semi-dense in $X$.

Note. Most of the results in this paper were stated without proof, or with only brief indications of proof in some cases, by the author in his Presidential Address before the American Mathematical Society [6]. 
For further discussion of these results, in particular for cases in which alternative dimension preserving forms of (4.2) above are possible, see [6].

5. Differentiable functions. We now show that a mapping from a region of the $z$-plane $Z$ into the $w$-plane $W$ generated by a function $w=f(z)$ satisfying certain differentiability conditions will satisfy the requirements needed in the preceding sections to insure the preservation of non-density for compact sets.

(5.1) Theorem. Let $w=f(z)$ be continuous in a region $X$ of $Z$ and differentiable at all points of a dense set $f^{-1}\left(Y_{0}\right)$ in $X$ which is the inverse of an open subset $Y_{0}$ of $Y=f(X)$. Then $f$ is strongly quasi-open, no component of a point inverse lying inside a closed 2-cell on $X$ separates $X$ and the set $f\left(L_{f}\right)$ is dense in $Y$. Further, $L_{f}$ is semi-dense in $X$.

Proof. (Note. In the proof of all but the final statement use is made of only easily established topological properties of functions meeting minimum differentiability requirements. In proving the last one, however, we use the property, rather more difficult to establish topologically, that a non-constant function everywhere differentiable in a region $R$ cannot be constant on any open set in $R$.)

To prove $f$ strongly quasi-open it suffices (see $\S 7$ of [6]) to show that for any elementary region $R$ in $X$ with boundary $C$ in $X$,

( $\left.^{*}\right) f(R+C)=f(C)$ the union of bounded components of $W-f(C)$,

where "elementary" means that $R$ is bounded and $C$ consists of a finite number of disjoint simple closed curves. To accomplish this, let $S$ be a component of $W-f(C)$ such that the set $S_{0}=S \cdot f(R)$ is not empty. Since $R \cdot f^{-1}\left(S_{0}\right)$ is open and nonempty, it therefore intersects $f^{-1}\left(Y_{0}\right)$. Thus $S_{0} \cdot Y_{0}$ is not empty. Let $Q$ be a component of $S_{0} \cdot Y_{0}$. Since $R \cdot f^{-1}(Q)$ is open and thus has only a countable number of components, there exists a component $T$ of $R \cdot f^{-1}(Q)$ on which $f$ is not constant. As $f$ is differentiable on $T$ by hypothesis [because $T \subset f^{-1}\left(Y_{0}\right)$ ] there exists a point $z_{0} \in T$ where $f^{\prime}\left(z_{0}\right) \neq 0$. Now using properties of the circulation index, it readily follows that $Q$ contains the interior of a square and thus contains a point $q$ such that $f^{\prime}(z) \neq 0$ for all $z \in f^{-1}(q)$. Since this makes the circulation index equal $2 \pi i$ times a positive integer when taken around any sufficiently small circle enclosing a point of $f^{-1}(q)$, it results at once that the circulation index taken over all of $C$ of $f$ about $q$ must be $\neq 0$. Further, since this latter index is constant throughout $S$, that is, it has the same value when any $p \in S$ is substituted for $q$, it follows that every point $p$ of $S$ must belong to $f(R)$. For details of the argument needed here using the circulation index the reader is referred to the last paragraph of $\S 5$ of [7]. 
Hence we have $S \subset f(R)$. This gives $\left({ }^{*}\right)$, however, because $f(R+C)$ obviously cannot contain the whole unbounded component of $W-f(C)$. Thus any component of $W-f(C)$ intersecting $f(R)$ must be bounded and must lie wholly in $f(R)$. Accordingly $f$ is strongly quasi-open.

Suppose, contrary to the second assertion, that some component $K$ of $f^{-1}\left(w_{0}\right)$, for some $w_{0} \in Y$, lies inside a closed 2-cell $A$ on $X$ and separates $X$. Then one component $Q$ of $X-K$ must lie wholly inside $A$ since only one component of $X-K$ intersects the edge of $A$. Let $y$ be a point of $f(Q+K)$ such that $\left|y-w_{0}\right|=\max \left|f(z)-w_{0}\right|$ for $z \in Q+K$. Then $Q$ contains a component $H$ of $f^{-1}(y)$ and $H$ is compact. Accordingly, by the strong quasi-openness of $f, y$ must be interior to $f(Q)$ contrary to $\left|f(z)-w_{0}\right| \leqq\left|y-w_{0}\right|$ for all $z \in Q$.

That $f\left(L_{f}\right)$ is dense in $Y$ is an immediate consequence of the fact that $Y_{0}$ is dense in $Y$ and the quasi-openness of $f$ already established. For any open set in $Y$ thus contains the interior $I$ of a square such that $f$ is differentiable everywhere on $f^{-1}(I)$. Thus for some $q \in I$, $f^{\prime}(z) \neq 0$ for all $z \in f^{-1}(q)$. This makes $f^{-1}(q)$ a scattered set which therefore surely lies in $L_{f}$.

Finally, to prove $L_{f}$ semi-dense in $X$ we note first that if $T$ is any region in $X$ on which $f$ is non-constant and everywhere differentiable, then as shown above in the second paragraph of this proof, $T$ contains a point $z_{0}$ where $f^{\prime}\left(z_{0}\right) \neq 0$ and indeed $f(T)$ contains points $q$ such that $f^{\prime}(z)$ does not vanish on $f^{-1}(q)$, so that $f^{-1}(q) \subset L_{f}$. Accordingly any such region $T$ intersects $L_{f}$. Now if $U$ is any open set in $X$ with an open image, $f(U) \cdot Y_{0}$ contains a region $Q$ and if $T$ is any component of $f^{-1}(Q) \cdot U$ on which $f$ is not constant (and there are such components $T$ because the collection of all components of $f^{-1}(Q) \cdot U$ is countable), then $T$ intersects $L_{f}$ as shown above. However, the same holds for an arbitrary subregion $T_{0}$ of $T$, because $f$ is likewise non-constant and everywhere differentiable on $T_{0}$. Thus $L_{f}$ is dense in $T$.

\section{REFERENCES}

1. P. S. Alexandroff, Dokl. (C. R.) Akad. Nauk SSSR. IV (XIII), No. 7 (111), 295-299.

2. R. L. Moore, Concerning upper semi-continuous collections of continua, Trans. Amer. Math. Soc., 27 (1925), 416-428.

3. A. D. Wallace, Quasi-monotone transformations, Duke Math. J., 7 (1940), 136-145.

4. G. T. Whyburn, Irterior transformations on surfaces, Amer. J. Math., 60 (1938), 477-490.

5. - - An open mapping approach to Hurwitz's theorem, Trans. Amer Math. Soc., 71 (1951), 114.

6. _- Topological analysis, Bull. Amer. Math. Soc., 62 (1956), 204-218.

7. - Lectures on functions of a complex variable, Univ. of Michigan Press (1955), $1-14$. 



\section{PACIFIC JOURNAL OF MATHEMATICS}

\section{EDITORS}

H. L. ROYDEN

Stanford University

Stanford, California

R. A. Beaumont

University of Washington

Seattle 5, Washington

\section{A. L. Whiteman}

University of Southern California

Los Angeles 7, California

E. G. Straus

University of California

Los Angeles 24, California

\section{ASSOCIATE EDITORS}
E. F. BECKENBACH
C. E. BURGESS
M. HALL
E. HEWITT

\author{
A. HORN \\ V. GANAPATHY IYER \\ R. D. JAMES \\ M. S. KNEBELMAN
}

L. NACHBIN

I. NIVEN

G. SZEKERES

T. G. OSTROM

M. M. SCHIFFER
F. WOLF

K. YOSIDA

\section{SUPPORTING INSTITUTIONS}

\author{
UNIVERSITY OF BRITISH COLUMBIA \\ CALIFORNIA INSTITUTE OF TECHNOLOGY \\ UNIVERSITY OF CALIFORNIA \\ MONTANA STATE UNIVERSITY \\ UNIVERSITY OF NEVADA \\ OREGON STATE COLLEGE \\ UNIVERSITY OF OREGON \\ UNIVERSITY OF SOUTHERN CALIFORNIA
}

\author{
STANFORD UNIVERSITY \\ UNIVERSITY OF UTAH \\ WASHINGTON STATE COLLEGE \\ UNIVERSITY OF WASHINGTON \\ AMERICAN MATHEMATICAL SOCIETY \\ CALIFORNIA RESEARCH CORPORATION \\ HUGHES AIRCRAFT COMPANY \\ THE RAMO-WOOLDRIDGE CORPORATION
}

Mathematical papers intended for publication in the Pacific Journal of Mathematics should be typewritten (double spaced), and the author should keep a complete copy. Manuscripts may be sent to any of the editors. All other communications to the editors should be addressed to the managing editor, E. G. Straus at the University of California, Los Angeles 24, California.

50 reprints per author of each article are furnished free of charge; additional copies may be obtained at cost in multiples of 50 .

The Pacific Journal, of Mathematics is published quarterly, in March, June, September, and December. The price per volume (4 numbers) is $\$ 12.00$; single issues, $\$ 3.50$. Back numbers are available. Special price to individual faculty members of supporting institutions and to individual members of the American Mathematical Society: $\$ 4.00$ per volume; single issues, $\$ 1.25$.

Subscriptions, orders for back numbers, and changes of address should be sent to Pacific Journal of Mathematics, 2120 Oxford Street, Berkeley 4, California.

Printed at Kokusai Bunken Insatsusha (International Academic Printing Co., Ltd.), No. 10, 1-chome, Fujimi-cho, Chiyoda-ku, Tokyo, Japan.

PUBLISHED BY PACIFIC JOURNAL OF MATHEMATICS, A NON-PROFIT CORPORATION

The Supporting Institutions listed above contribute to the cost of publication of this Journal, but they are not owners or publishers and have no responsibility for its content or policies. 


\section{Pacific Journal of Mathematics}

\section{Vol. 7, No. 2 \\ February, 1957}

William F. Donoghue, Jr., The lattice of invariant subspaces of a completely continuous quasi-nilpotent transformation ................... 1031

Michael (Mihály) Fekete and J. L. Walsh, Asymptotic behavior of restricted extremal polynomials and of their zeros.................... 1037

Shaul Foguel, Biorthogonal systems in Banach spaces ............... 1065

David Gale, A theorem on flows in networks ................... 1073

Ioan M. James, On spaces with a multiplication .................. 1083

Richard Vincent Kadison and Isadore Manual Singer, Three test problems in operator theory .................................... 1101

Maurice Kennedy, A convergence theorem for a certain class of Markoff processes........................................ 1107

G. Kurepa, On a new reciprocity, distribution and duality law ........ 1125

Richard Kenneth Lashof, Lie algebras of locally compact groups ........ 1145

Calvin T. Long, Note on normal numbers .................... 1163

M. Mikolás, On certain sums generating the Dedekind sums and their reciprocity laws ..................................... 1167

Barrett O'Neill, Induced homology homomorphisms for set-valued maps......................................... 1179

Mary Ellen Rudin, A topological characterization of sets of real numbers........................................... 1185

M. Schiffer, The Fredholm eigen values of plane domains 1187

F. A. Valentine, A three point convexity property .........

Alexander Doniphan Wallace, The center of a compact lattice is totally

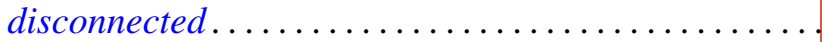

Alexander Doniphan Wallace, Two theorems on topological lattices.

G. T. Whyburn, Dimension and non-density preservation of mappings...

John Hunter Williamson, On the functional representation of certain algebraic systems ... 